\title{
Density functional study of electronic and optical properties of ternary mixed chalcogenides topological insulators
}

\begin{abstract}
This paper presented a theoretical study of structural, electronic, and optical properties of the ternary mixed chalcogenides Topological Insulators with a formula $\mathrm{M}_{2} \mathrm{X}_{2} \mathrm{Y}(\mathrm{M}=\mathrm{Bi}, \mathrm{X}=\mathrm{Te}$ and $\mathrm{Y}=\mathrm{Se}, \mathrm{S}$ ) using density functional theory (DFT) within the local density approximation (LDA). From the calculation, we have evaluated the bulk modulus and its corresponding pressure derivatives of these compounds. The linear photon-energy dependent of dielectric functions, some optical properties such as reflectivity, refraction index, conductivity function, and energy-loss spectra, have also been obtained and analyzed within the electronic band structures and density of states of these compounds.
\end{abstract}

Keyword: Density functional theory; electronic properties; Optical properties; ternary mixed chalcogenides 